\title{
A genetic variant in the promoter of IncRNA MALAT1 is related to susceptibility of ischemic stroke
}

\author{
Yan Wang ${ }^{1,2+}, X_{i-X i ~ G u}{ }^{1 \dagger}$, Hua-Tuo Huang ${ }^{2}$, Chun-Hong Liu² and Ye-Sheng Wei ${ }^{1,2^{*}}$
}

\begin{abstract}
Background: Metastasis-associated lung adenocarcinoma transcript-1 (MALAT1) was aberrantly expressed in diverse diseases. Particularly in ischemic stroke (IS), the abnormal expression of MALAT1 played important roles including promotion of angiogenesis, inhibition of apoptosis and inflammation and regulation of autophagy. However, the effects of genetic variation (single nucleotide polymorphisms, SNPS) of MALAT1 on IS have rarely been explored. This study aimed to investigate whether SNPs in promoter of MALAT1 were associated with the susceptibility to IS.

Methods: A total of 316 IS patients and 320 age-, gender-, and ethnicity-matched controls were enrolled in this study. Four polymorphisms in the promoter of MALAT1 (i.e., rs600231, rs1194338, rs4102217, and rs591291) were genotyped by using a custom-by-design 48-Plex SNPscan kit.

Results: The rs1194338 C > A variant in the promoter of MALAT1 was associated with the risk of IS (AC vs. CC: adjusted $\mathrm{OR}=0.623,95 \% \mathrm{Cl}, 0.417-0.932, P=0.021$; AA vs. $C \mathrm{C}$ : adjusted $\mathrm{OR}=0.474,95 \% \mathrm{Cl}, 0.226-0.991, P=0.047$; Dominant model: adjusted $\mathrm{OR}=0.596,95 \% \mathrm{Cl}, 0.406-0.874, P=0.008$; $\mathrm{A}$ vs. $\mathrm{C}$ adjusted $\mathrm{OR}=0.658,95 \% \mathrm{Cl}, 0.487-$ $0.890, P=0.007$ ). The haplotype analysis showed that rs600231-rs1194338-rs4102217-rs591291 (A-C-G-C) had a 1.3fold increased risk of IS (95\% Cl, 1.029-1.644, $P=0.027)$. Logistic regression analysis identified some independent impact factors for IS including rs1194338 AC/AA, TC, TG, HDL-C, LDL-C, Apo-A1, Apo-B and NEFA $(P<0.05)$.
\end{abstract}

Conclusions: These results suggest that the rs1194338 AC/AA genotypes may be a protective factor for IS.

Keywords: Ischemic stroke, Polymorphism, Metastasis associated lung adenocarcinoma transcript 1

\section{Background}

Stroke is a nervous system disease caused by the blood circulation disorder in the brain. It has a high mortality, disability and recurrence rate worldwide. Stroke has become the major cause of death in China, along with approximately 2.5 million new cases and 1.5 million deaths each year [1,2]. The ischemic stroke (IS) accounts for about $87 \%$ of total cases [3]. Therefore, it is urgently

\footnotetext{
* Correspondence: yeshengwei22@163.com

${ }^{\dagger}$ Yan Wang and Xi-Xi Gu contributed equally to this work.

'Department of Clinical Laboratory, The Affiliated Hospital of Guilin Medical

University, Lequn Road No.15, Guilin 541001, Guangxi Province, China

${ }^{2}$ Department of Clinical Laboratory, The Affiliated Hospital of Youjiang

Medical University for Nationalities, Baise, Guangxi, China
}

required to explore etiology for meaningful targets. As we all known, the IS was a multifactorial complex disease. Traditional factors such as age, gender, obesity, diabetes, hypertension and smoking can only explain part of the IS risk [4-7]. Twins, familial aggregation and genome-wide association studies revealed that heredity was also a powerful factor in progression of IS [8-12].

Long non-coding RNAs (lncRNAs), with a length of more than 200 nucleotides, are emerging as key regulators of physiological and pathological processes [13]. As a highly conserved lncRNA in mammals, MALAT1 played an important role in cancer. Ghafouri-Fard $\mathrm{S}$ et al. revealed that MALAT1 altered activity or functions 
of multiple cancer-related signaling pathways such as EMT and PI3K/AKT, but the role of MALAT1 in carcinogenesis was indefinite, which might be linked with cancer types and sub-types. Moreover, MALAT1 SNPs modulated the risk of cancer by possibly affecting function or expression levels [14]. Recently, increasing evidence has emerged to support the role of this lncRNA in IS. Using RNA sequencing technology, a lot of lncRNAs with abnormal expression were detected after $16 \mathrm{~h}$ under oxygen-glucose deprivation (OGD) condition [15]. MALAT1, one of the most highly up-regulated lncRNAs, was further confirmed to promote angiogenesis and autophagy, and reduce apoptosis and inflammation both in vitro and in vivo [16-18]. For example, MALAT1 reduced ischemic cerebral damages by regulating 15LOX1, VEGF and STAT3 related to angiogenesis [16]. Moreover, MALAT1 also acted as a competing endogenous RNA for miR-26b to directly up-regulate the expression of autophagy factor ULK2 to reduce the injury of brain microvascular endothelial cells [17]. Silencing of MALAT1 obviously increased expression of the proapoptotic and proinflammatory cytokines including Bim, IL-6, MCP-1 and E-selectin [18]. Taken together, MALAT1 appears important not only in cancer development but also in the progression of IS, but the exact mechanism is still not fully elucidated.

Currently, SNPs of IncRNAs have been verified to be associated with IS susceptibility, such as the rs 217727 $\mathrm{C}>\mathrm{T}$ and rs4929984 $\mathrm{C}>\mathrm{A}$ in lncRNA $H 19$ and the rs2240183 in promoter of lncRNA TUG1 [19-21]. It was reported that genetic variants in the promoter region could affect the expression, subcellular localization and structure stability, ultimately affecting progression of relevant diseases [22]. At the same time, studies also showed that MALAT1 SNPs affected the susceptibility and progression of diseases including hepatocellular cancer, lung adenocarcinoma and pulmonary arterial hypertension [23-25]. However, the impacts of MALAT1 SNPs on IS is rarely explored. To our knowledge, no study was conducted for association between the SNPs (rs600231, rs1194338, rs591291 and rs4102217) in promoter of MALAT1 and IS risk. Given the above, we performed SNPs analysis of MALAT1 from 636 samples (320 controls and 316 IS patients) in Chinese southwestern population, attempting to identify new candidates for the etiology of IS.

\section{Methods}

\section{Study population}

A total of 316 patients with IS and 320 controls were consecutively recruited from the Affiliated Hospital of Youjiang Medical University for Nationalities, Guangxi, China, between March 2015 and July 2018. All of the subjects were native people living in Guangxi province who were unrelated to Han Chinese. The IS patients were diagnosed based on clinical manifestations, physical examination and cranial computed tomography or magnetic resonance imaging. Exclusion criteria were as follows: hemorrhagic stroke, craniocerebral trauma, cardiogenic thrombosis and tumors. Controls matched the cases at the age, and gender frequency were selected from the hospital's health care center during the same period. Individuals with autoimmune diseases, liver diseases, genetic diseases, blood disorders and tumors were excluded. The clinical data such as age, gender, hypertension, diabetes, smoking status, total cholesterol (TC), triglyceride (TG), high-density lipoprotein cholesterol (HDL-C), low-density lipoprotein cholesterol (LDL-C), apolipoprotein A1 (Apo-A1), apolipoprotein B (Apo-B) and non-esterified fatty acid (NEFA) were collected from our medical records. The interval time was within $24 \mathrm{~h}$ between IS onset and biochemical test.

\section{SNPs selection}

(I) The selection criteria for SNP are as follows: (i) tagSNPs in lncRNA MALAT1; (ii) the SNPs in promoter region of MALAT1; (iii) in silico analysis predicted potentially functional SNPs in the promoter region of MALAT1; (iv) the frequency of minor allele $>5 \%$ in Chinese Han population. (v) the functional SNPs have been identified in published literature. Finally, four SNPs of rs600231 A>G, rs1194338 C>A, rs4102217 G>C and rs591291 $\mathrm{C}>\mathrm{T}$ were selected for further analysis.

\section{Genotyping}

Genomic DNA was extracted from mononuclear cells of peripheral blood by a salting-out method. About 3-5 ml blood samples was taken into ethylene diamine tetraacetic acid tubes from each subject before treatment. Genotyping was performed on an ABI 3500 Genetic Analyzer (Applied Biosystems, CA, USA) using the custom-bydesign 48-Plex SNPscan kit (Genesky Biotechnologies Inc., Shanghai, China). Genotypic primers for rs600231, rs1194338, rs4102217and rs591291 were showed in Table 1. Meanwhile, about $10 \%$ of all samples were selected at random for Sanger sequencing and reached a $100 \%$ consistent rate.

\section{Statistical analysis}

The Student's t-test was used to compare continuous data (Mean \pm SD) such as clinical data from IS cases and controls. The chi-squared test was chosen to analyze Hardy-Weinberg equilibrium (HWE) and categorical data (proportions) such as sex, hypertension, diabetes mellitus and smoker data. Logistic regression was used to assess the risk of IS by odds ratios (OR), 95\% confidence intervals (CIs) and $P$ value after adjustment of age, gender, diabetes mellitus, hypertension, smoker, TC, 
Table 1 The primer sequences used for detecting four SNPS of the MALAT1

\begin{tabular}{llll}
\hline SNPs & allele A1 & allele A2 & universal primer \\
\hline rs600231 & 5'-TGAAACCCAGCAGACAGGACT-3' & 5'-TGAAACCCAGCAGACAGGACC-3' & 5'-GTCACTTCACAGAGAGCTGAGGGC-3' \\
rs1194338 & 5'-GGCTCCAGGGCCGTAGATCAA-3' & 5'-GGCTCCAGGGCCGTAGATCAC-3' & 5'-GGATCTCTCAGAAGCTTGTCTCTTGA-3' \\
rs4102217 & 5'-CCTGCTGCCTCCCTTCCTGTG-3' $^{\prime}$ ' & 5'-CCTGCTGCCTCCCTTCCTGTC-3' & 5'-CAGCACTTCTGTCAGTCTCTCCAA-3' \\
rs591291 & 5'-CCCTCACCCCCGGGTCTGTG-3' & 5'-CCCTCACCCCCGGGTCTGTA-3' & 5'-GAACCTGTATCCATGGCTTGTITT-3' \\
\hline
\end{tabular}

SNPs Single nucleotide polymorphisms

TG, HDL-C, LDL-C, Apo-A1, Apo-B and NEFA. Linkage disequilibrium (LD) and haplotype analysis were carried out by SHEsis software (http://analysis.bio-x.cn/ myAnalysis.php). The SPSS statistical software package version 20.0 (SPSS, Chicago, USA) was used for all of the statistical analysis. The $P<0.05$ was considered statistically significant.

\section{Results}

\section{Characteristics of the study population}

The results are exhibited in Table 2. No significant difference was observed in distributions of age and gender between cases and controls. The frequencies of hypertension, diabetes mellitus and smoker in IS patients were obviously higher than those in controls $(P<0.05)$. In addition, IS patients displayed higher levels of TC, TG, LDL-C and Apo-B, and lower levels of HDL-C, Apo-A1, and NEFA (all $P<0.05$ ).

\section{Association between MALAT1 polymorphisms and IS risk}

The analysis of MALAT1 SNPs for IS risk is revealed in Table 3. The genotype distributions in controls conformed to HWE $(P=0.780$ for rs600231, $P=0.858$ for rs1194338, $P=0.569$ for rs4102217, $P=0.582$ for rs591291). Among these SNPs of MALAT1, the

Table 2 Clinical characteristics of the study population

\begin{tabular}{llll}
\hline Variables & Controls, $n=320$ & IS patients, $n=316$ & $P$ value \\
\hline Age, years (Mean \pm SD) & $60.72 \pm 10.77$ & $62.23 \pm 11.36$ & 0.087 \\
Gender (M / F) & $204 / 116$ & $216 / 100$ & 0.220 \\
Hypertension (\%) & $59(18.4 \%)$ & $126(39.9)$ & $<\mathbf{0 . 0 0 1}$ \\
Diabetes mellitus (\%) & $30(9.4 \%)$ & $50(15.8 \%)$ & $<\mathbf{0 . 0 0 1}$ \\
Smoker (\%) & $50(15.6 \%)$ & $98(31.0 \%)$ & $<\mathbf{0 . 0 0 1}$ \\
TCH (mmol/L) & $4.19 \pm 0.79$ & $4.64 \pm 1.16$ & $<\mathbf{0 . 0 0 1}$ \\
TG $(\mathrm{mmol} / \mathrm{L})$ & $1.37 \pm 0.99$ & $1.81 \pm 1.31$ & $<\mathbf{0 . 0 0 1}$ \\
HDL-C (mmol/L) & $1.50 \pm 0.31$ & $1.13 \pm 0.32$ & $<\mathbf{0 . 0 0 1}$ \\
LDL $-\mathrm{C}(\mathrm{mmol} / \mathrm{L})$ & $2.39 \pm 0.66$ & $2.92 \pm 0.98$ & $<\mathbf{0 . 0 0 1}$ \\
Apo-A1(g/L) & $1.73 \pm 1.10$ & $1.23 \pm 0.26$ & $<\mathbf{0 . 0 0 1}$ \\
Apo-B (g/L) & $0.77 \pm 0.31$ & $1.00 \pm 0.31$ & $<\mathbf{0 . 0 0 1}$ \\
NEFA (mmol/L) & $0.71 \pm 0.30$ & $0.53 \pm 0.28$ & $<\mathbf{0 . 0 0 1}$ \\
\hline
\end{tabular}

IS ischemic stroke, SD Standard deviation, M Male, F Female, TC Total cholesterol, TG Triglyceride, HDL-C High density lipoprotein-cholesterol, $L D L-C$ Low density lipoprotein-cholesterol, Apo-A1 Apolipoprotein A1, Apo-B Apolipoprotein B, NEFA Non-esterified fatty acid
Table 3 Association between the MALAT1 polymorphisms and risk of IS

\begin{tabular}{|c|c|c|c|c|}
\hline SNPS & $\begin{array}{l}\text { Controls } \\
n=320(n \%)\end{array}$ & $\begin{array}{l}\text { IS patient } \\
n=316(n \%)\end{array}$ & AORt (95\%Cl) & $P+$ value \\
\hline \multicolumn{5}{|l|}{ rs600231 } \\
\hline AA & 117 (36.6) & $120(38.0)$ & 1.000 (ref) & \\
\hline$A G$ & $151(47.2)$ & $154(48.7)$ & $0.987(0.651-1.496)$ & 0.950 \\
\hline GG & $52(16.2)$ & $42(13.3)$ & $0.778(0.434-1.395)$ & 0.399 \\
\hline Dominant & & & $0.933(0.629-1.386)$ & 0.733 \\
\hline Recessive & & & $1.276(0.750-2.171)$ & 0.369 \\
\hline A & $385(60.2)$ & $394(62.3)$ & 1.000 (ref) & \\
\hline G & $255(39.8)$ & $238(37.7)$ & $0.906(0.688-1.192)$ & 0.481 \\
\hline \multicolumn{5}{|l|}{ rs1194338 } \\
\hline CC & $154(48.1)$ & $188(59.5)$ & 1.000 (ref) & \\
\hline$A C$ & $135(42.2)$ & $106(33.5)$ & $0.623(0.417-0.932)$ & 0.021 \\
\hline AA & $31(9.7)$ & $22(7.0)$ & $0.474(0.226-0.991)$ & 0.047 \\
\hline Dominant & & & $0.596(0.406-0.874)$ & 0.008 \\
\hline Recessive & & & $1.721(0.841-3.523)$ & 0.137 \\
\hline C & $443(69.2)$ & $482(76.3)$ & 1.000 (ref) & \\
\hline A & $197(30.8)$ & $150(23.7)$ & $0.658(0.487-0.890)$ & 0.007 \\
\hline \multicolumn{5}{|l|}{ rs4102217 } \\
\hline GG & $243(75.9)$ & $237(75.0)$ & 1.000 (ref) & \\
\hline$C G$ & $73(22.8)$ & $69(21.8)$ & $1.186(0.753-1.868)$ & 0.463 \\
\hline $\mathrm{CC}$ & $4(1.3)$ & $10(3.2)$ & $2.322(0.605-8.906)$ & 0.219 \\
\hline Dominant & & & $1.254(0.807-1.947)$ & 0.314 \\
\hline Recessive & & & $0.452(0.119-1.721)$ & 0.244 \\
\hline G & $559(87.3)$ & $543(85.9)$ & 1.000 (ref) & \\
\hline C & $81(12.7)$ & $89(14.1)$ & $1.280(0.869-1.886)$ & 0.212 \\
\hline \multicolumn{5}{|l|}{ rs591291 } \\
\hline $\mathrm{CC}$ & $123(38.5)$ & $129(40.8)$ & 1.000 (ref) & \\
\hline$C T$ & $147(45.9)$ & $144(45.6)$ & $0.916(0.607-1.384)$ & 0.678 \\
\hline$\pi$ & $50(15.6)$ & $43(13.6)$ & $0.752(0.421-1.343)$ & 0.336 \\
\hline Dominant & & & $0.873(0.592-1.289)$ & 0.495 \\
\hline Recessive & & & $1.268(0.743-2.163)$ & 0.384 \\
\hline C & $393(61.4)$ & $402(63.6)$ & 1.000 (ref) & \\
\hline T & 247 (38.6) & $230(36.4)$ & $0.877(0.665-1.155)$ & 0.350 \\
\hline
\end{tabular}

IS Ischemic stroke, OR Odds ratio, 95\% Cl, 95\% confidence interval, + Adjusted by age, gender, hypertension, diabetes mellitus, smoker, TCH, TG, HDL-C, LDLC, Apo-A1, Apo-B, NEFA 
rs1194338 AC, AA and AC/AA genotype decreased the risk of IS with adjusted OR of $0.623,0.474$ and 0.596 , respectively (AC vs. CC: $95 \% \mathrm{CI}, 0.417-0.932, P=0.021$; AA vs. CC: $95 \%$ CI, $0.226-0.991, P=0.047$; AC/AA vs. CC: $95 \% \mathrm{CI}, 0.406-0.874, P=0.008)$. Similarly, the risk of IS in carriers with A allele was lower than $\mathrm{C}$ allele (AOR $=0.658,95 \% \mathrm{CI}, 0.487-0.890, P=0.007$ ). No significant association was found between other SNPs (rs600231, rs4102217, rs591291) and IS risk.

Haplotype analysis of MALAT1 polymorphisms with IS risk To further estimate the association between MALAT1 polymorphism and risk of IS, we performed haplotype analysis. The analysis showed that there was linkage disequilibrium among the four loci, among which the rs600231 and rs591291 showed a strong linkage disequilibrium ( $\left.D^{\prime}=0.94, r^{2}=0.83\right)$. As summarized in Table 4, possible four haplotypes were listed, and the rs600231rs1194338-rs4102217-rs591291 (A-C-G-C) haplotype had a 1.3-fold increased risk of IS (95\% CI, 1.029-1.644, $P=0.027$ ).

\section{Multiple logistic regression analysis}

As shown in Table 5, the rs1194338 AC/AA affected the IS risk together with blood lipid index. The specific data were as follows: $\mathrm{TC}(\mathrm{OR}=1.607 ; 95 \% \mathrm{CI}, 1.356-1.903)$, TG $(\mathrm{OR}=1.482 ; 95 \% \mathrm{CI}, 1.242-1.770)$, HDL-C $(\mathrm{OR}=$ 0.020; 95\%CI, 0.010-0.040), LDL-C (OR = 2.181; 95\%CI, 1.764-2.697), Apo-A1 (OR = 0.006; 95\%CI, 0.002-0.013), Apo-B $\quad(\mathrm{OR}=23.315 ; \quad 95 \% \mathrm{CI}, \quad 11.576-46.959)$, NEFA $(\mathrm{OR}=0.092 ; 95 \% \mathrm{CI}, 0.048-0.177)$ and $\mathrm{rs} 1194338 \mathrm{AC} / \mathrm{AA}$ $(\mathrm{OR}=0.632 ; 95 \% \mathrm{CI}, 0.461-0.865)($ all $p<0.05)$.

\section{The analysis of MALAT1 SNPs and blood lipid levels}

The association between MALAT1 SNPs and lipid levels is showed in Table 6. Unfortunately, no evidence of association was observed between SNPs of MALAT1 and clinical blood lipid levels of IS patients $(P>0.05)$.

\section{Bioinformatics analysis}

GTEx data (https://www.gtexportal.org/home/) was used to identify correlations between SNPs and tissue-specific gene expression levels. The analysis of expression quantitative trait loci (eQTL) showed the rs1194338 SNPs

Table 4 Haplotype analysis of the MALAT1 polymorphisms with risk of IS

\begin{tabular}{lllll}
\hline Haplotype & Controls $(\mathrm{n} \%)$ & IS $(\mathrm{n} \%)$ & OR $(95 \% \mathrm{Cl})$ & $P$ value \\
\hline ACGC & $351(54.9)$ & $373(59.1)$ & $1.301(1.029-1.644)$ & $\mathbf{0 . 0 2 7}$ \\
GAGT & $91(15.3)$ & $86(13.6)$ & $0.903(0.659-1.238)$ & 0.527 \\
GACT & $71(11.1)$ & $57(9.1)$ & $0.822(0.569-1.187)$ & 0.296 \\
GCGT & $61(9.6)$ & $62(9.9)$ & $1.076(0.742-1.561)$ & 0.699 \\
\hline
\end{tabular}

IS Ischemic stroke, OR Odds ratio, 95\% Cl 95\% confidence interval. Only frequency greater than $1 \%$ is listed
Table $\mathbf{5}$ Logistic regression analysis for independent factors of IS susceptibility

\begin{tabular}{lllll}
\hline Variables & B & S.E & $P$ value & OR $(95 \% \mathrm{Cl})$ \\
\hline TC & 0.47 & 0.086 & $<\mathbf{0 . 0 0 1}$ & $1.607(1.356-1.903)$ \\
TG & 0.39 & 0.090 & $<\mathbf{0 . 0 0 1}$ & $1.482(1.242-1.770)$ \\
HDL-C & -3.89 & 0.345 & $<\mathbf{0 . 0 0 1}$ & $0.020(0.010-0.040)$ \\
LDL-C & 0.78 & 0.108 & $<\mathbf{0 . 0 0 1}$ & $2.181(1.764-2.697)$ \\
Apo-A1 & -5.193 & 0.429 & $<\mathbf{0 . 0 0 1}$ & $0.006(0.002-0.013)$ \\
Apo-B & 3.149 & 0.357 & $<\mathbf{0 . 0 0 1}$ & $23.315(11.576-46.959)$ \\
NEFA & -2.389 & 0.334 & $<\mathbf{0 . 0 0 1}$ & $0.092(0.048-0.177)$ \\
rs1194338AC/AA & -0.459 & 0.160 & $\mathbf{0 . 0 0 4}$ & $0.632(0.461-0.865)$ \\
\hline TCToL chos
\end{tabular}

TC Total cholesterol, TG Triglyceride, HDL-C High density lipoproteincholesterol, LDL-C Low density lipoprotein-cholesterol, Apo-A1 Apolipoprotein A1, Apo-B Apolipoprotein B, NEFA Non-esterified fatty acid

were associated with expression of MALAT1 in single tissue (Fig. 1a), and the carriers with rs1194338 AA increased expression of MALAT1 in single brain tissue such as brain-hippocampus, brain-cerebellar hemisphere (Fig. 1b-c) $(P<0.001)$.

\section{Discussion}

In the current study, we explored the association between SNPs in the promoter of MALAT1 and risk of IS. Significant differences were observed in the distribution of the rs1194338 AC/AA genotype and A allele between controls and cases. Further analysis showed that MALAT1 rs1194338 A allele, AA, AC genotype and the dominant model were associated with decreased risk of IS. Regression analysis revealed that rs1194338AC/AA was one of impact factors of IS together with the lipid index such as TC, TG, HDL-C. In addition, haplotype analysis showed that the ACGC haplotype increased 1.3fold risk of IS. These findings implicate that MALAT1 rs1194338 may played the role in the etiology of IS.

MALAT1, an $8.1 \mathrm{~kb}$ IncRNA, located on human chromosome 11q13. In 2003, Ji et al. first discovered and named MALAT1 from lung cancer cells [26]. Subsequently, MALAT1 was confirmed to be associated with tumors cell proliferation, metastasis, survival, and recurrence [27]. New evidence showed that MALAT1 was abundantly expressed in vascular endothelial cells and participated in processes of neuroprotection of IS by improvement of cognitive function, promotion of angiogenesis, inhibition of apoptosis and inflammation, regulation of autophagy, and protection of blood-brain barrier function [16-18, 28, 29]. The PI3K/AKT pathway was implicated in cell proliferation, apoptosis, and survival under physiological and pathological conditions [30]. The previous study showed a neuroprotective role of early activation of PI3K in ischemic stroke [31]. The result from Yuan et al. demonstrated that overexpression of MALAT1 decreased cell apoptosis by activating of 
Table 6 Association between the MALAT1 SNPs and blood lipid levels in IS

\begin{tabular}{|c|c|c|c|c|c|c|c|}
\hline SNPS & $\begin{array}{l}\mathrm{TC} \\
\mathrm{mmol} / \mathrm{L}\end{array}$ & $\begin{array}{l}\mathrm{TG}, \\
\mathrm{mmol} / \mathrm{L}\end{array}$ & $\begin{array}{l}\mathrm{HDL}-\mathrm{C} \\
\mathrm{mmol} / \mathrm{L}\end{array}$ & $\begin{array}{l}\mathrm{LDL}-\mathrm{C} \\
\mathrm{mmol} / \mathrm{L}\end{array}$ & $\begin{array}{l}\text { Apo-A1, } \\
\text { g/L }\end{array}$ & $\begin{array}{l}\text { Apo-B, } \\
\text { g/L }\end{array}$ & $\begin{array}{l}\text { NEFA, } \\
\mathrm{mmol} / \mathrm{L}\end{array}$ \\
\hline \multicolumn{8}{|l|}{ rs600231 } \\
\hline AA & $4.65 \pm 1.20$ & $1.79 \pm 1.34$ & $1.13 \pm .33$ & $2.94 \pm 1.03$ & $1.23 \pm 0.26$ & $1.00 \pm 0.32$ & $0.53 \pm 0.28$ \\
\hline$A G / G G$ & $4.64 \pm 1.08$ & $1.83 \pm 1.27$ & $1.12 \pm .31$ & $2.88 \pm 0.90$ & $1.23 \pm 0.25$ & $1.00 \pm 0.30$ & $0.55 \pm 0.28$ \\
\hline $\mathrm{t}$ & 0.090 & -0.245 & 0.384 & 0.462 & -0.138 & 0.007 & -0.620 \\
\hline$p$ & 0.928 & 0.806 & 0.701 & 0.645 & 0.890 & 0.995 & 0.535 \\
\hline \multicolumn{8}{|l|}{ rs1194338 } \\
\hline CC & $4.68 \pm 1.11$ & $1.79 \pm 1.38$ & $1.14 \pm 0.32$ & $2.94 \pm 0.97$ & $1.23 \pm 0.23$ & $0.99 \pm 0.30$ & $0.52 \pm 0.27$ \\
\hline AC/AA & $4.62 \pm 1.19$ & $1.82 \pm 1.27$ & $1.12 \pm 0.32$ & $2.90 \pm 0.99$ & $1.23 \pm 0.28$ & $1.01 \pm 0.31$ & $0.54 \pm 0.29$ \\
\hline $\mathrm{t}$ & 0.478 & -0.170 & 0.519 & 0.434 & -0.032 & -0.382 & 0.498 \\
\hline$p$ & 0.633 & 0.865 & 0.604 & 0.664 & 0.974 & 0.703 & 0.619 \\
\hline \multicolumn{8}{|l|}{ rs4102217 } \\
\hline GG & $4.52 \pm 1.21$ & $1.72 \pm 1.20$ & $1.12 \pm 0.30$ & $2.80 \pm 0.99$ & $1.21 \pm 0.26$ & $0.98 \pm 0.30$ & $0.55 \pm 0.29$ \\
\hline $\mathrm{CG} / \mathrm{CC}$ & $4.68 \pm 1.14$ & $1.83 \pm 1.35$ & $1.13 \pm 0.33$ & $2.96 \pm .98$ & $1.24 \pm 0.26$ & $1.01 \pm 0.31$ & $0.53 \pm 0.28$ \\
\hline $\mathrm{t}$ & -1.079 & -0.677 & -0.374 & -1.274 & -0.642 & -0.861 & 0.562 \\
\hline$p$ & 0.281 & 0.499 & 0.708 & 0.204 & 0.522 & 0.390 & 0.574 \\
\hline \multicolumn{8}{|l|}{ rs591291 } \\
\hline CC & $4.64 \pm 1.22$ & $1.82 \pm 1.40$ & $1.13 \pm 0.33$ & $2.93 \pm 1.05$ & $1.23 \pm 0.27$ & $1.00 \pm 0.32$ & $0.53 \pm 0.28$ \\
\hline $\mathrm{CT} / \mathrm{TT}$ & $4.63 \pm 1.07$ & $1.78 \pm 1.19$ & $1.12 \pm 0.31$ & $2.89 \pm 0.88$ & $1.24 \pm 0.25$ & $1.00 \pm 0.29$ & $0.54 \pm 0.28$ \\
\hline $\mathrm{t}$ & 0.072 & 0.262 & 0.124 & 0.460 & -0.447 & 0.083 & -0.550 \\
\hline p & 0.943 & 0.794 & 0.902 & 0.646 & 0.655 & 0.934 & 0.583 \\
\hline
\end{tabular}

TC Total cholesterol, TG Triglyceride, HDL-C High density lipoprotein-cholesterol, LDL-C Low density lipoprotein-cholesterol, Apo-A1 Apolipoprotein A1, Apo-B, Apolipoprotein B, NEFA Non-esterified fatty acid

PI3K/AKT pathway, eventually protect human cerebrovascular endothelial cells in OGD and reoxygenation condition [32]. The above indicate that the MALAT1 plays a critical role in ischemic stroke, and its high expression may contribute to the protection against brain injury.

In recent years, the association between lncRNA related polymorphisms and risk of diseases has become hotpot of research. As a lncRNA with a wide range of functions, MALAT1 related SNPs attracted the attention of researchers. For instance, Eftekharian et al. explored the relationship between two MALAT1 SNPs (rs619586 and rs3200401) and multiple sclerosis (MS) in an Iranian population, and confirmed the $\mathrm{G}$ allele of rs619586 significantly reduced the risk of MS with OR of 0.65 [33]. This means that functional SNPs of MALAT1 may serve as a potential indicator for relevant diseases. The ischemic stroke is one of the diseases threatening human health, and the pathogenesis of IS remain to be fully understood. Actually, increasing studies focused on SNPs of lncRNA involved in process of IS. For example, the rs2240183 C allele of lncRNA TUG1 was associated with a higher risk of IS by possibly binding to GATA-1 and elevating TUG1 levels [19]. The lncRNA ANRIL rs2383207 increased the risk of IS by 1.52 -fold under the recessive model [20]. Furthermore, the rs $217727 \mathrm{TT}$ and rs4929984 AA in the lncRNA H19 increased the risk of IS, with adjusted OR of 4.288, 3.020, respectively [21]. Those studies provided a new perspective on the genetic mechanism of IS. Given above, we hypothesized that the MALAT1 SNPs were associated with IS risk. Our results supported the above assumption. As shown in Table 3, case-control studies indicated the rs1194338 A allele, $\mathrm{AC}$ and AA genotype of MALAT1 contributed to decreasing risk of IS. Additionally, the ACAG haplotype increased risk of IS (Table 4). Logistic regression also manifested the effect of the rs1194338 AC/AA on risk of IS (Table 5).

The rs1194338, a functional site, located in the promoter region of the MALAT1. Recently, several studies indicated the relationship between rs1194338 variant and human diseases. In hepatocellular carcinoma (HCC), female patients and patients with a smoking habit who carried the CA + AA genotype of rs1194338 had a lower risk of developing vascular invasion and a higher ChildPugh grade, respectively [34]. This suggested there was an interactive function between rs1194338 and the environment. Whether the rs1194338 interacts with the environment in IS remains to be further explored. In colorectal cancer, previous studies found carriers with 


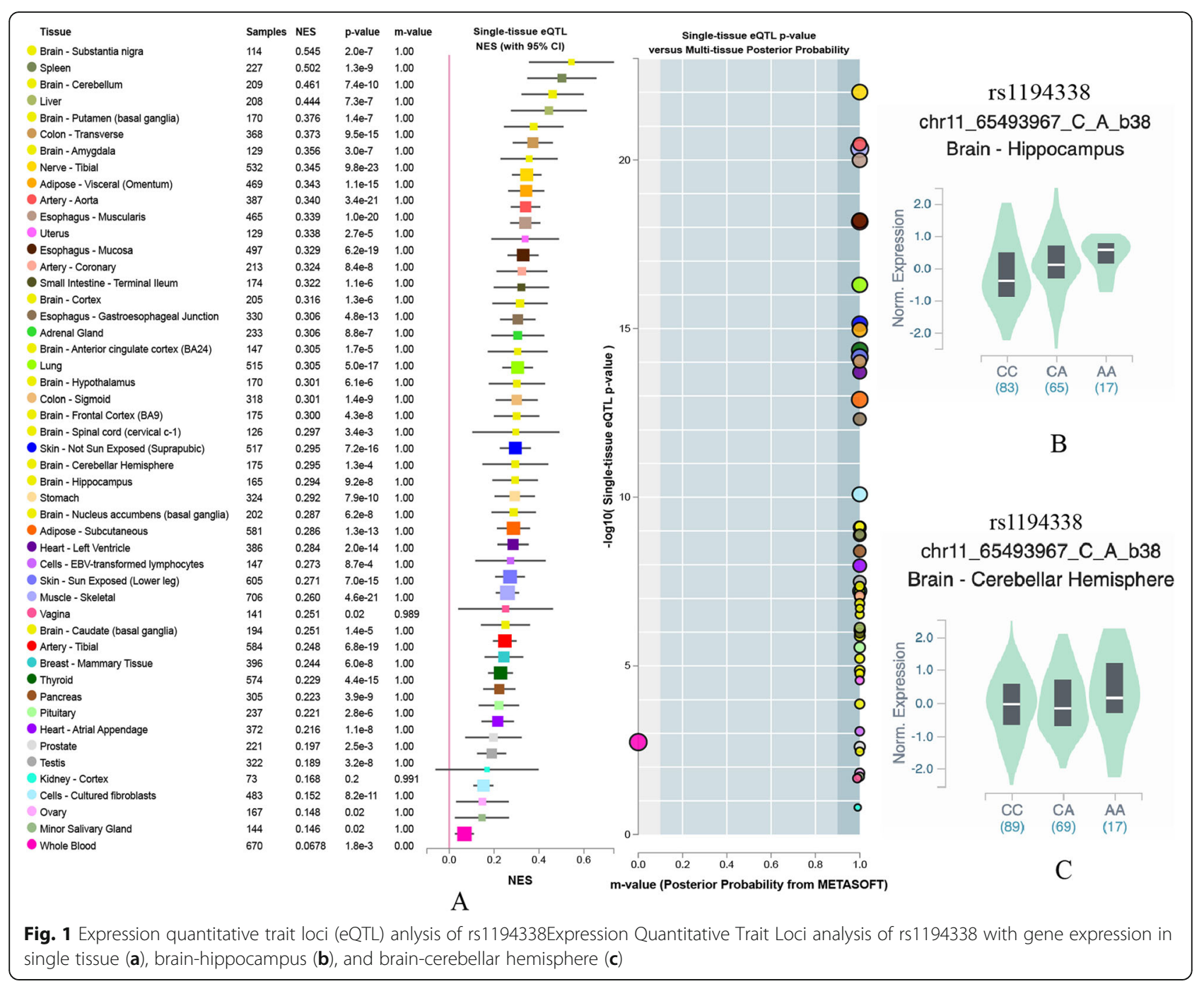

$\mathrm{AA}$ and AC genotype of the rs1194338 were lower risk than CC genotype, and the conclusion from Li's study showed no statistically significant difference in expression of MALAT1 between CC and AA genotype at rs1194338 [35, 36]. However, the GTEx database showed rs1194338 SNPs had differences in expression of MALAT1. Particularly, the AA genotype of rs1194338 significantly increased expression of MALAT1 compared to the CC genotype in brain-hippocampus and cerebellar hemisphere tissues $(P<0.001)$ (Fig. 1). Based on the above background, we speculated that the rs1194338 AC/AA genotype might increase the expression of MALAT1, which activated related pathways such as PI3K/AKT, thereby decreasing the risk of IS. Further studies are needed to investigate the correlation between the rs1194338 SNPs and expression of MALAT1 and the precise mechanism of rs1194338 SNPs in IS.

To our knowledge, the study of rs600231 A > G variant with risk of diseases have been rarely reported, but rs4102217 and rs591291 SNPs were evaluated in diseases.
Zhang et al. indicated rs4102217 and rs591291 SNPs were not associated with susceptibility of rheumatoid arthritis [37]. The Study of association with HCC have shown rs4102217 had a 1.32-fold risk in the dominant model, and rs591291 highlighted better prognosis in female and HBV negative subgroups, but association between MALAT1 haplotype (rs4102217-rs591291-rs11227209rs619586) and HCC risk were not observed [23]. In our study, we found that the ACAG haplotype had a 1.3-fold risk of IS although SNPs (rs600231, rs4102217, rs591291) did not correlate with the susceptibility of IS. It is well known that alteration in blood lipid levels is one of the risk factors in atherosclerotic plaques formation. Atherosclerotic plaques caused easily hypoxia, and possibly resulted in severe diseases such as ischemic stroke [38]. According to the report, MALAT1 was involved in lipid metabolism [39]. Thus, we further analyzed the relationship between the SNPs of MALAT1 and blood lipid levels in IS patients. Unfortunately, we failed to observe the significant association. These findings will help improve our 
understanding of the role of MALAT1 genetic variants in the pathogenesis of IS.

Although the results we got were promising, limitations still remained. Firstly, a relatively small sample may limit the authenticity of the statistical analysis. Secondly, our study is hospital-based case-control study, and potential selection bias may exist. In addition, the population we studied came from the southwest of China. There are distribution differences in polymorphisms of the same locus among different races according to the 1000 Genomes Project Data. Therefore, larger sample sizes from other medical centers of different races and ethnicities are needed to further confirm the role of MALAT1 SNPs in IS susceptibility. Finally, the effects of MALAT1 SNPs on IS are very interesting, but its mechanism is unclear. Both RNA and DNA should be collected simultaneously from the same samples to further verify the effects of SNPs on expression of MALAT1.

\section{Conclusions}

In conclusion, our study provides a link between rs1194338 SNPs in promoter of MALAT1 and the risk of IS, helping to explore the potential molecular mechanisms of IS. In the future, large-scale samples study can be performed among different populations.

\section{Abbreviations \\ Apo-A1: Apolipoprotein A1; ApoB: Apolipoprotein B; Cls: Confidence intervals; eQTL: Expression Quantitative Trait Loci; F: Female; HCC: Hepatocellular carcinoma; HDL-C: High-density lipoprotein cholesterol; HWE: Hardy-Weinberg equilibrium; IS: Ischemic stroke; LD: Linkage disequilibrium; LDL-C: Low-density lipoprotein cholesterol; IncRNAs: Iong non-coding RNAs; M: Male; MALAT1: Metastasis-associated lung adenocarcinoma transcript-1; MS: Multiple sclerosis; NEFA: Non-esterified fatty acid; OGD: Oxygen-glucose deprivation; OR: Odds ratio; SD: Standard deviation; SNP: Single nucleotide polymorphism; TC: Total cholesterol; TG: Triglyceride}

\section{Acknowledgements}

We are deeply grateful to all participants of this study.

\section{Authors' contributions}

WYS guided and revised manuscript. WY and GXX participated in experiment and wrote the manuscript. HHT helped to perform experiments. LCH performed the statistical analysis and figures. All authors reviewed the manuscript. The authors read and approved the final manuscript.

\section{Funding}

This work was supported by the Natural Science Foundation of China, Grant/ Award Number: 81560552; Natural Science Foundation of Guangxi, China, Grant/Award Number: 2018GXNSFAA138120; Key Research Projects of Guangxi, China, Grant/Award Number: 2018AB58018.

\section{Availability of data and materials}

The datasets supporting the conclusions of this article are included within the article.

\section{Ethics approval and consent to participate}

The procedure was reviewed and approved by the Institutional Ethical Committee of the Affiliated Hospital of Youjiang Medical University for Nationalities, in accordance with the principles of the Helsinki Declaration. Each subject signed informed consent and agreed to make the data public.
Consent for publication

Not applicable.

\section{Competing interests}

The authors declare that they have no competing interests.

Received: 9 January 2020 Accepted: 13 March 2020

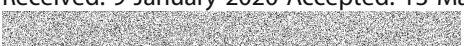

\section{References}

1. Zhou M, Wang H, Zeng X, Yin P, Zhu J, Chen W, Li X, Wang L, Wang L, Liu $Y$, et al. Mortality, morbidity, and risk factors in China and its provinces, 1990-2017: a systematic analysis for the global burden of disease study 2017. Lancet. 2019:394:1145-58.

2. Liu L, Wang D, Wong KSL, Wang Y. Stroke and stroke care in China. Stroke. 2011:42:3651-4

3. Benjamin EJ, Muntner P, Alonso A, Bittencourt MS, Callaway CW, Carson AP, Chamberlain AM, Chang AR, Cheng S, Das SR, et al. Heart disease and stroke statistics-2019 update: a report from the American Heart Association. Circulation. 2019;139:e56-28.

4. Larsson SC, Burgess S, Micha LK. Smoking and stroke: a mendelian randomization study. Ann Neurol. 2019;86:468-71.

5. Lee JS, Chang PY, Zhang Y, Kizer JR, Best LG, Howard BV. Triglyceride and HDL-C dyslipidemia and risks of coronary heart disease and ischemic stroke by glycemic dysregulation status: the strong heart study. Diabetes Care. 2017:40:529-37.

6. Yong H, Foody J, Linong J, Dong Z, Wang Y, Ma L, Meng HJ, Shiff S, Dayi H. A systematic literature review of risk factors for stroke in China. Cardiol Rev. 2013;21:77-93.

7. Fahimfar N, Khalili D, Mohebi R, Azizi F, Hadaegh F. Risk factors for ischemic stroke; results from 9 years of follow-up in a population based cohort of Iran. BMC Neurol. 2012;12:117.

8. Anderson CD, Biffi A, Rost NS, Cortellini L, Furie KL, Rosand J. Chromosome 9p21 in ischemic stroke: population structure and meta-analysis. Stroke. 2010;41:1123-31.

9. Hsu WC, Chen ST, Wu YR, Chang HS, Lyu RK, Lo LS. The association of stroke and family history of stroke depends on its subtypes and gender: a family history study in Taiwan. Acta Neurol Taiwanica. 2009;18:161-9.

10. Ikram MA, Seshadri S, Bis JC, Fornage M, DeStefano AL, Aulchenko YS, Debette S, Lumley T, Folsom AR, van den Herik EG, et al. Genomewide association studies of stroke. N Engl J Med. 2009;360:1718-28.

11. Jood K, Ladenvall C, Rosengren A, Blomstrand C, Jern C. Family history in ischemic stroke before 70 years of age. Stroke. 2005;36:1383-7.

12. Bak S, Gaist D, Sindrup SH, Skytthe A, Christensen K. Genetic liability in stroke: a long-term follow-up study of Danish twins. Stroke. 2002;33:769-74.

13. Ulitsky I, Bartel DP. lincRNAs: genomics, evolution, and mechanisms. Cell. 2013;154:26-46.

14. Ghafouri-Fard S, Ashrafi Hafez A, Taheri M. Metastasis associated lung adenocarcinoma transcript 1: an update on expression pattern and functions in carcinogenesis. Exp Mol Pathol. 2020;112:104330.

15. Zhang J, Yuan L, Zhang X, Hamblin MH, Zhu T, Meng F, Li Y, Chen YE, Yin $\mathrm{KJ}$. Altered long non-coding RNA transcriptomic profiles in brain microvascular endothelium after cerebral ischemia. Exp Neurol. 2016;277: 162-70.

16. Wang C, Qu Y, Suo R, Zhu Y. Long non-coding RNA MALAT1 regulates angiogenesis following oxygen-glucose deprivation/reoxygenation. J Cell Mol Med. 2019;23:2970-83.

17. Li Z, Li J, Tang N. Long noncoding RNA Malat1 is a potent autophagy inducer protecting brain microvascular endothelial cells against oxygenglucose deprivation/reoxygenation-induced injury by sponging miR-26b and upregulating ULK2 expression. Neuroscience. 2017;354:1-10.

18. Zhang X, Tang X, Liu K, Hamblin MH, Yin KJ. Long noncoding RNA Malat1 regulates cerebrovascular pathologies in ischemic stroke. J Neurosci. 2017; 37:1797-806.

19. Wei YS, Yang J, He YL, Shi X, Zeng ZN. A functional polymorphism in the promoter of TUG1 is associated with an increased risk of ischemic stroke. J Cell Mol Med. 2019;23:6173-81.

20. Yang J, Gu L, Guo X, Huang J, Chen Z, Huang G, Kang Y, Zhang X, Long J, Su L. LnCRNA ANRIL expression and ANRIL gene polymorphisms contribute to the risk of ischemic stroke in the Chinese Han population. Cell Mol Neurobiol. 2018;38:1253-69. 
21. Wang J, Cao B, Han D, Sun M, Feng J. Long non-coding RNA H19 induces cerebral ischemia reperfusion injury via activation of autophagy. Aging Dis. 2017;8:71.

22. Deng $\mathrm{N}$, Zhou $H$, Fan $H$, Yuan $\mathrm{Y}$. Single nucleotide polymorphisms and cancer susceptibility. Oncotarget. 2017;8:110635-49.

23. Wang BG, Xu Q, Lv Z, Fang XX, Ding HX, Wen J, Yuan Y. Association of twelve polymorphisms in three onco-IncRNA genes with hepatocellular cancer risk and prognosis: a case-control study. World J Gastroenterol. 2018; 24:2482-90.

24. Wang JZ, Xiang JJ, Wu LG, Bai YS, Chen ZW, Yin XQ, Wang Q, Guo WH, Peng $Y$, Guo $\mathrm{H}$, et al. A genetic variant in long non-coding RNA MALAT1 associated with survival outcome among patients with advanced lung adenocarcinoma: a survival cohort analysis. BMC Cancer. 2017;17:167.

25. Zhuo Y, Zeng Q, Zhang P, Li G, Xie Q, Cheng Y. Functional polymorphism of IncRNA MALAT1 contributes to pulmonary arterial hypertension susceptibility in Chinese people. Clin Chem Lab Med. 2017;55:38-46.

26. Ji P, Diederichs S, Wang W, Böing S, Metzger R, Schneider PM, Tidow N, Brandt B, Buerger $\mathrm{H}$, Bulk E, et al. MALAT-1, a novel noncoding RNA, and thymosin beta4 predict metastasis and survival in early-stage non-small cell lung cancer. Oncogene. 2003;22:8031-41.

27. Arun G, Diermeier S, Akerman M, Chang KC, Wilkinson JE, Hearn S, Kim Y, MacLeod AR, Krainer AR, Norton L, et al. Differentiation of mammary tumors and reduction in metastasis upon Malat1 IncRNA loss. Genes Dev. 2016;30: 34-51.

28. Patel NA, Moss LD, Lee JY, Tajiri N, Acosta S, Hudson C, Parag S, Cooper DR, Borlongan CV, Bickford PC. Long noncoding RNA MALAT1 in exosomes drives regenerative function and modulates inflammation-linked networks following traumatic brain injury. J Neuroinflammation. 2018;15:204.

29. Puthanveetil P, Chen S, Feng B, Gautam A, Chakrabarti S. Long non-coding RNA MALAT1 regulates hyperglycaemia induced inflammatory process in the endothelial cells. J Cell Mol Med. 2015;19:1418-25.

30. Dudek H, Datta SR, Franke TF, Birnbaum MJ, Yao R, Cooper GM, Segal RA, Kaplan DR, Greenberg ME. Regulation of neuronal survival by the serinethreonine protein kinase Akt. Science. 1997;275:661-5.

31. Kim YS, Yoo A, Son JW, Kim HY, Lee YJ, Hwang S, Lee KY, Lee YJ, Ayata C, Kim HH, et al. Early activation of phosphatidylinositol 3-kinase after ischemic stroke reduces infarct volume and improves long-term behavior. Mol Neurobiol. 2017;54:5375-84.

32. Wang $G$, WU Y, Zhu Y. Mechanism of MALAT1 preventing apoptosis of vascular endothelial cells induced by oxygen-glucose deficiency and reoxidation. Artif Cells Nanomed Biotechnol. 2018;46:798-805.

33. Eftekharian MM, Noroozi R, Komaki A, Mazdeh M, Ghafouri-Fard S, Taheri M. MALAT1 genomic variants and risk of multiple sclerosis. Immunol Investig. 2019:48:549-54

34. Yuan LT, Chang JH, Lee HL, Yang YC, Su SC, Lin CL, Yang SF, Chien MH. Genetic variants of IncRNA MALAT1 exert diverse impacts on the risk and clinicopathologic characteristics of patients with hepatocellular carcinoma. J Clin Med. 2019:8:1406. https://doi.org/10.3390/jcm8091406.

35. Zhao K, Jin S, Wei B, Cao S, Xiong Z. Association study of genetic variation of IncRNA MALAT1 with carcinogenesis of colorectal cancer. Cancer Manag Res. 2018;10:6257-61.

36. Li Y, Bao C, Gu S, Ye D, Jing F, Fan C, Jin M, Chen K. Associations between novel genetic variants in the promoter region of MALAT1 and risk of colorectal cancer. Oncotarget. 2017;8:92604-14.

37. Zhang TP, Zhu BQ, Tao SS, Fan YG, Li XM, Pan HF, Ye DQ. Long non-coding RNAs genes polymorphisms and their expression levels in patients with rheumatoid arthritis. Front Immunol. 2019;10:2529.

38. Tell GS, Crouse JR, Furberg CD. Relation between blood lipids, lipoproteins, and cerebrovascular atherosclerosis. A review. Stroke. 1988:19:423-30.

39. Mongelli A, Martelli F, Farsetti A, Gaetano C. The dark that matters: Long non-coding RNAs as master regulators of cellular metabolism in noncommunicable diseases. Front Physiol. 2019;10:369.

\section{Publisher's Note}

Springer Nature remains neutral with regard to jurisdictional claims in published maps and institutional affiliations.

\section{Ready to submit your research? Choose BMC and benefit from:}

- fast, convenient online submission

- thorough peer review by experienced researchers in your field

- rapid publication on acceptance

- support for research data, including large and complex data types

- gold Open Access which fosters wider collaboration and increased citations

- maximum visibility for your research: over $100 \mathrm{M}$ website views per year

At BMC, research is always in progress.

Learn more biomedcentral.com/submissions 